\title{
Adaptive Multi-Stream Relaying
}

\author{
Furuzan Atay Onat and \\ Halim Yanikomeroglu \\ Dept. of SCE, Carleton University, \\ Ottawa, Canada \\ e-mail: $\{$ furuzan, halim\} @ sce.carleton.ca
}

\author{
Shalini Periyalwar \\ Independent Consultant \\ Ottawa, Canada \\ e-mail: speriyalwar@hotmail.com
}

\begin{abstract}
In this paper, we study a cooperative network with one source-destination pair and a relay assisting their communication. All terminals have multiple antennas, which enables them to transmit multiple streams simultaneously. Both the source and the relay use spatial multiplexing to transmit while the receivers at the relay and the destination are linear MMSE receivers.

We extend some of the well known digital relaying protocols to multi-stream case and also propose two new adaptive protocols to reduce error propagation at the relay. We evaluate the block error rate of all the protocols and show the benefit of stream by stream decisions at the relay.
\end{abstract}

\section{INTRODUCTION}

A significant increase in capacity of wireless channels is possible by deploying multiple antennas at both transmitter and receiver sides if the channel has rich scattering [1], [2]. Even practical architectures with certain constraints like VBlast [3], can bring much higher spectral efficiencies than the conventional systems by spatial multiplexing at the transmitter side. Multiple antennas either at the transmitter or the receiver side can also improve link reliability through space time coding [4] or receive diversity.

In this paper, our focus is on transmitter architectures that use spatial multiplexing. Since we plan to generalize our model to a network of independent single antenna sources in the future, we avoid coding across the transmit antennas. Hence, transmit diversity through space-time coding at the source is not available. In linear MIMO receivers, such as $\mathrm{ZF}$ and MMSE filters, diversity order of each streams is $N-M+1$, where $M$ is the number of transmit antennas and $N$ is the number of receive antennas. This might not be high enough when the receiver does not have additional antennas. In such cases, we propose to use relaying to improve the diversity of MIMO links. We analyze a system where the source and destination communicate with the largest multiplexing gain possible $(N=M)$ and the relay is the main source of diversity.

Relay protocols in the literature can be classified as analog relaying and digital relaying. In analog relaying the relay terminal amplifies the received signal and then retransmits. One disadvantage of analog relaying is the noise and interference enhancement. In digital relaying the relay detects and decodes the source signal and then regenerates and retransmits it. This protocol requires more processing at the relay compared to analog relaying.

This work was supported by Wireless Tech. Labs, Nortel Networks, Ottawa.
In addition to working as traditional "repeaters" to decrease effective path-loss, if the destination terminal is capable of combining direct signal and the signals from relay nodes, relays can induce diversity against multi-path fading. In [5], [6] users acting as relays were shown to induce spatial diversity in the system. Diversity obtained by users relaying each other's data is usually referred as cooperative diversity. Protocols that exploit this kind of diversity are called as diversity relaying protocols.

Instead of users acting as relays for each other, it is also possible to use fixed relays that are deployed as a part of the infrastructure. These relays, unlike mobile users, can be connected to the power line. Hence, transmit power is not an issue for these terminals. Moreover, they can extend the battery life of mobile users by taking over some of their power burden.

In digital diversity relaying, if the relay transmits a data block that has detection/decoding errors, it is likely that there will be a decoding error at the destination. This problem, usually called error propagation, limits the diversity order of digital relaying [5], [7], [8]. However, selective protocols that allow the relay to transmit only when it can decode the source signal reliably can be designed [5], [9], [10]. This way error propagation can be significantly reduced and digital relaying can provide full diversity order.

The work in the literature on relaying schemes is mostly confined to single antenna case. Relaying of multiple streams is a relatively new problem, which is recently considered in [11], [12]. These references consider only the so called conventional relaying, where the destination make use of only the relayed signal. They ignore the direct link between the source and the destination either due to shadowing or high attenuation because of large distance between the two terminals. Although, the effect of this typically weaker link can be small in terms of capacity, our preliminary results indicate that in Rayleigh fading environments it is quite useful in terms of diversity even for linear topologies, which cause the maximum source-destination separation. Shadowing in the direct link, on the other, can diminish the advantage of combining the direct signal and the relayed signal. However, in a general model where all three links have independent shadowing, which is not studied here, we expect that diversity relaying will still outperform conventional relaying.

We consider block based digital relaying protocols to assist the spatial multiplexing of $M$ streams. We study fixed and adaptive protocols, which rely on error detection mechanisms at the relay. In Non-Adaptive Digital Relaying (NADR) protocol, the relay always retransmits all the streams in the second 


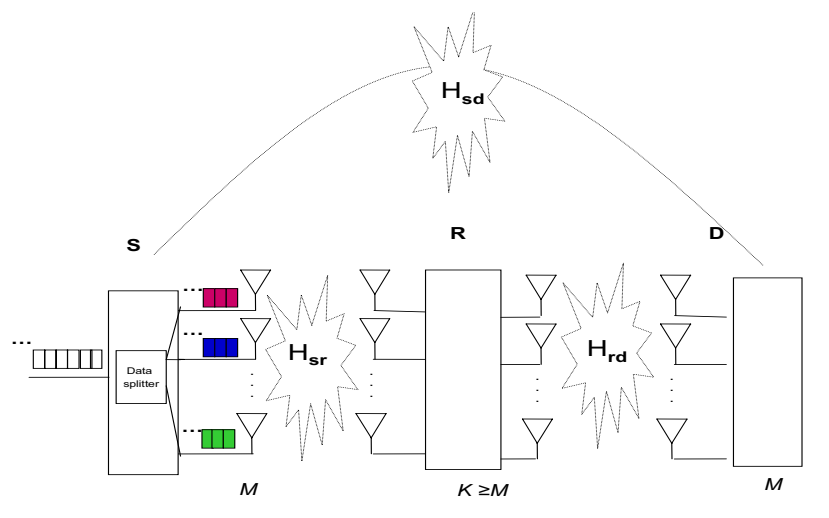

Fig. 1.

slot. In, Adaptive Digital Relaying (ADR), the relay transmits only if it does not detect any errors in any of the streams. In general, the relay might have correctly detected only $k<M$ of all the streams. Stream Adaptive Digital Relaying (SADR) protocol takes advantage of such cases by allowing the relay transmit only these $k$ streams. This way, the time slot allocated for the relay is used efficiently and at the same time error propagation is prevented. All the relaying protocols detailed in the paper operate in two equal time slots. To enable fair comparison with direct transmission, we also present a simple protocol with no relay cooperation called Time-Division Direct Transmission (TDDT) protocol.

\section{A. Notation}

Superscripts $T$ and $H$ are used for transpose and Hermitian conjugate of matrices, respectively. $\operatorname{diag}\left\{x_{1}, x_{2}, \ldots, x_{n}\right\}$ stands for an $n \times n$ diagonal matrix with given elements on its diagonal. $\mathbf{I}_{n}$ and $\mathbf{0}_{m, n}$ denote the $n \times n$ identity matrix and $m \times n$ zero matrix, respectively. A circularly symmetric complex Gaussian vector with zero mean and identity covariance matrix is called a standard Gaussian random vector. $\mathbf{H}_{\mathbf{w}}(N, M)$ denotes an $N \times M$ random matrix, whose columns are i.i.d standard Gaussian random vectors. $a \sim b$ means that random variable $a$ is distributed as $b$.

\section{SYSTEM MODEL}

We consider a three terminal network as shown in Fig. 1. The source and the destination have $M$ antennas each, while the relay has $(K \geq M)$ antennas. We call such a system as an $M \times K \times M$ system. When the relay has more antennas than the streams it is required to transmit, it chooses those antennas at random. Source-Relay, RelayDestination and Source-Destination channels are represented by matrices, $\mathbf{H}_{\mathbf{s r}} \in \mathbb{C}^{K \times M}, \mathbf{H}_{\mathbf{r d}} \in \mathbb{C}^{M \times M}$, and $\mathbf{H}_{\mathbf{s d}} \in$ $\mathbb{C}^{M \times M}$, respectively. We assume independent block fading, where all the channel matrices stay the same for $2 T$ symbol periods and then they all change to new states, which are independent from each other and their previous states. The channel state information (CSI) is available only at the receiver side for all three links. All channel states assumed to follow
Rayleigh fading. Hence, we denote:

$$
\begin{aligned}
\mathbf{H}_{\mathbf{s r}} & \sim \sqrt{g_{s r} \frac{E_{s}}{M N_{0}}} \mathbf{H}_{\mathbf{w}}(K, M) \\
\mathbf{H}_{\mathbf{r d}} & \sim \sqrt{g_{r d} \frac{E_{r}}{M N_{0}}} \mathbf{H}_{\mathbf{w}}(M, M) \\
\mathbf{H}_{\mathbf{s d}} & \sim \sqrt{g_{s d} \frac{E_{s}}{M N_{0}}} \mathbf{H}_{\mathbf{w}}(M, M)
\end{aligned}
$$

where $E_{s}$ and $E_{r}$ are the transmit energy per symbol duration at the source and the relay, respectively. Gains of the three channels are denoted by $g_{s r}, g_{r d}$ and $g_{s d}$. $\mathbf{H}_{\mathrm{w}}$ 's represent i.i.d. Rayleigh fading.

All the relaying protocols we study here, assume a halfduplex relay. Hence, the transmissions of the source and the relay are arranged by time-division. The transmissions take place in two equal time slots, each having $T$ symbol periods. During the first time slot, the source transmits $M$ streams from $M$ transmit antennas simultaneously. Then, in the second slot, the relay transmits using at most $M$ antennas.For simplicity, we ignore the overhead due to error detection and signaling required for the protocols. The received signals at the first and second slots are given by:

$$
\begin{aligned}
\mathbf{y}_{\mathbf{r}}(n) & =\mathbf{H}_{\mathbf{s r}} \mathbf{x}(n)+\mathbf{n}_{\mathbf{r}}(n) \\
\mathbf{y}_{\mathbf{d} \mathbf{1}}(n) & =\mathbf{H}_{\mathbf{s d}} \mathbf{x}(n)+\mathbf{n}_{\mathbf{d} \mathbf{1}}(n) \\
\mathbf{y}_{\mathbf{d} \mathbf{2}}(n) & =\mathbf{H}_{\mathbf{s d}} \hat{\mathbf{x}}(n)+\mathbf{n}_{\mathbf{d} \mathbf{2}}(n)
\end{aligned}
$$

where $n=1,2, \ldots, T$ is the symbol index. The symbol vector detected by the relay is denoted by $\hat{x}$. Symbols are MPSK modulated and they are i.i.d. from time to time and from antenna to antenna. Hence, we can write $E\left[\mathbf{x}(n) \mathbf{x}^{H}(m)\right]=$ $\delta(n-m) \mathbf{I}_{M}$. In the rest of the paper, we drop symbol index to simplify notation. Both at the relay and the destination, we assume linear MMSE receivers. In the cases where the destination combines the direct signal and the relayed signal, it combines the signals in the MMSE sense.

\section{DiRECT TRANSMISSION}

Due to time-division, the relay protocols require twice the bandwidth needed by the direct transmission at fixed transmission rate. To be able to make fair comparison, we use the following version of direct transmission: The source divides its streams into two sets of equal size. Assuming $M$ is even, each set has $M / 2$ streams. In the first time slot, the first set of streams are transmitted from their assigned antennas and the second set follows in the second slot. Again, the assignment is random and one-to-one. That is each antenna is used only once. We call this protocol as Time Division Direct Transmission (TDDT). This system can be represented by:

$$
\mathbf{y}=\mathbf{H}_{\mathbf{e}} \mathbf{x}+\mathbf{n}
$$

where

$$
\mathbf{H}_{\mathbf{e}}=\sqrt{g_{s d} \frac{E_{s}}{M N_{0}}}\left[\begin{array}{cc}
\mathbf{A}_{\mathbf{1}} & \mathbf{0}_{M \times(M / 2)} \\
\mathbf{0}_{M \times(M / 2)} & \mathbf{A}_{\mathbf{2}}
\end{array}\right],
$$


$\mathbf{A}_{1}$ and $\mathbf{A}_{\mathbf{2}}$ are independent and $\mathbf{A}_{\mathbf{1}}, \mathbf{A}_{\mathbf{2}} \sim \mathbf{H}_{\mathbf{w}}(M, M / 2)$. At the destination the symbols are filtered using a bank of MMSE combiners, which is given by:

$$
\mathbf{W}=\left(\mathbf{H}_{\mathbf{e}}{ }^{H} \mathbf{H}_{\mathbf{e}}+N_{0} \mathbf{I}_{\mathbf{M}}\right)^{-1} \mathbf{H}_{\mathbf{e}}{ }^{H}
$$

Then, they are detected by slicing the decision variable vector $\hat{\mathbf{y}}=\mathbf{W y}$.

\section{Multi-Stream Relaying Protocols}

We consider three digital diversity relaying protocols: NonAdaptive DR (NADR), Adaptive DR (ADR) and Stream Adaptive DR (SADR). For comparison, we also evaluate the performance of Conventional DR (CDR) and direct transmission (DT) and TDDT protocols.

\section{A. Conventional Digital Relaying $(C D R)$}

In CDR, the relay detects $M \times T$ symbol block and retransmits it in the second slot without error detection. Then, the destination performs detection based only on this relay signal. Hence, the system is given by:

$$
\mathbf{y}_{\mathrm{d} 2}=\mathbf{H}_{\mathrm{rd}} \hat{\mathbf{x}}+\mathbf{n}_{\mathrm{d} 2}
$$

\section{B. Non-Adaptive Digital Relaying (NADR)}

Here, different from CDR, the destination combines the direct signal and the relay signal. If the relay has detected all the symbols correctly in the first slot $(\hat{\mathrm{x}}=\mathrm{x})$, from the destination's point on view, the system can be represented as:

$$
\left[\begin{array}{l}
\mathbf{y}_{\mathrm{d} 1} \\
\mathbf{y}_{\mathrm{d} 2}
\end{array}\right]=\left[\begin{array}{l}
\mathbf{H}_{\mathrm{sd}} \\
\mathbf{H}_{\mathrm{rd}}
\end{array}\right] \mathrm{x}+\left[\begin{array}{l}
\mathbf{n}_{\mathrm{d} 1} \\
\mathbf{n}_{\mathrm{d} 2}
\end{array}\right]
$$

Then, $\mathbf{W}$ is obtained based on the equivalent channel matrix $\mathbf{H}_{\mathbf{e}}$ as in (6), where:

$$
\begin{aligned}
\mathbf{H}_{\mathbf{e}} & =\left[\begin{array}{l}
\mathbf{H}_{\mathbf{s d}} \\
\mathbf{H}_{\mathbf{r d}}
\end{array}\right] \\
& \sim\left[\begin{array}{cc}
\sqrt{\frac{g_{s d} E_{s}}{M N_{0}}} \mathbf{I}_{M} & \mathbf{0}_{M \times M} \\
\mathbf{0}_{M \times M} & \sqrt{\frac{g_{r d} E_{r}}{M N_{0}}} \mathbf{I}_{M}
\end{array}\right] \mathbf{H}_{\mathbf{w}}(2 M, M)
\end{aligned}
$$

\section{Adaptive Digital Relaying (ADR)}

In this protocol, we assume an error detection code that operates on the whole space time block. The relay can detect any number errors but cannot identify at which streams these errors occurred. Hence, it retransmits only when all the streams are error-free. When the relay retransmits, the system can be represented by (7).

\section{Stream Adaptive Digital Relaying (SADR)}

In SADR, each stream has its own error detection symbols and the relay can decide if a stream has any errors or not. Let $k$ be the number of error-free streams. Without loss of generality, we assume that these streams have been transmitted from the antennas $1,2, \ldots, k$ of the source. Then the equivalent channel matrix is given by:

$$
\mathbf{H}_{\mathbf{e}}=\left[\begin{array}{cc}
\sqrt{g_{s d \frac{E_{s}}{M N_{0}}}} \mathbf{B}_{\mathbf{1}} & \sqrt{g_{s d} \frac{E_{s}}{M N_{0}}} \mathbf{C}_{\mathbf{1}} \\
\sqrt{g_{r d \frac{E_{r}}{M N_{0}}}} \mathbf{B}_{\mathbf{2}} & \mathbf{0}_{M \times(M-k)}
\end{array}\right]
$$

where $\mathbf{B}_{\mathbf{1}}, \mathbf{B}_{\mathbf{2}} \sim \mathbf{H}_{\mathbf{w}}(M, k), \mathbf{C}_{\mathbf{1}} \sim \mathbf{H}_{\mathbf{w}}(M, M-k)$.

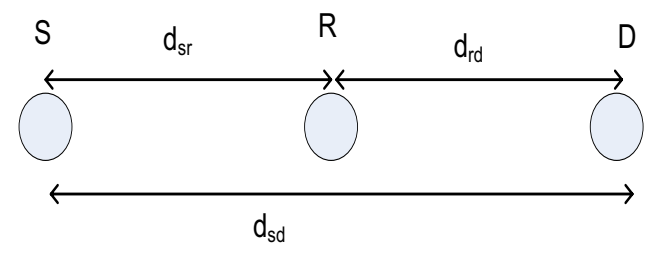

Fig. 2. Illustration of the linear topology

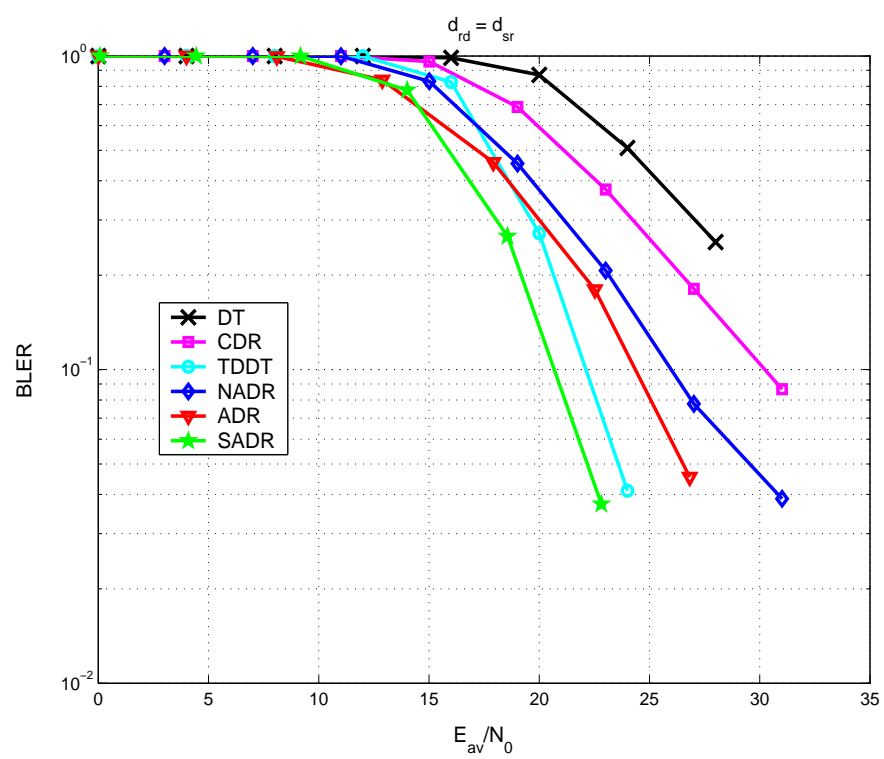

Fig. 3. Block error rates for topology $1, d_{r d}=d_{s r}$. Relative channel gains are $g_{s r}=0 d B, g_{r d}=0 d B$ and $g_{s d}=-9.03 d B$

\section{Numerical Results}

We note that the performances of relaying schemes usually depend on the average channel qualities, which are decided by network topology and terrain conditions. We assume that the source, the relay and the destination are located on a line as shown in Fig. 2. The distance between the source and the relay is normalized to 1 and the relative gains are determined based on path-loss with exponent $\alpha=3$. We consider three topologies, which result in different relative gains: $d_{r d}=1,2,5$.

We assume that the source and the relay transmit with the same power. Hence, $E_{s}=E_{r}=E$ for relaying protocols. For CDR and NADR the total average energy used per symbol is $E_{a v}=2 E / M$. However, for ADR and SDR the relay does not always transmit and $E_{a v} / E$ is not fixed for all $E$. Hence, for these protocols, we calculate $E_{a v}$ within the simulation and then we plot the results as a function of $E_{a v} / N_{0}$. Figs. 3-5 show the block error rate (BLER) for three different topologies calculated using Monte Carlo simulations. In all three figures, we observe that the benefit of conventional relaying is rather limited compared to all other diversity relaying schemes. We note that linear topology maximizes the direct link distance 


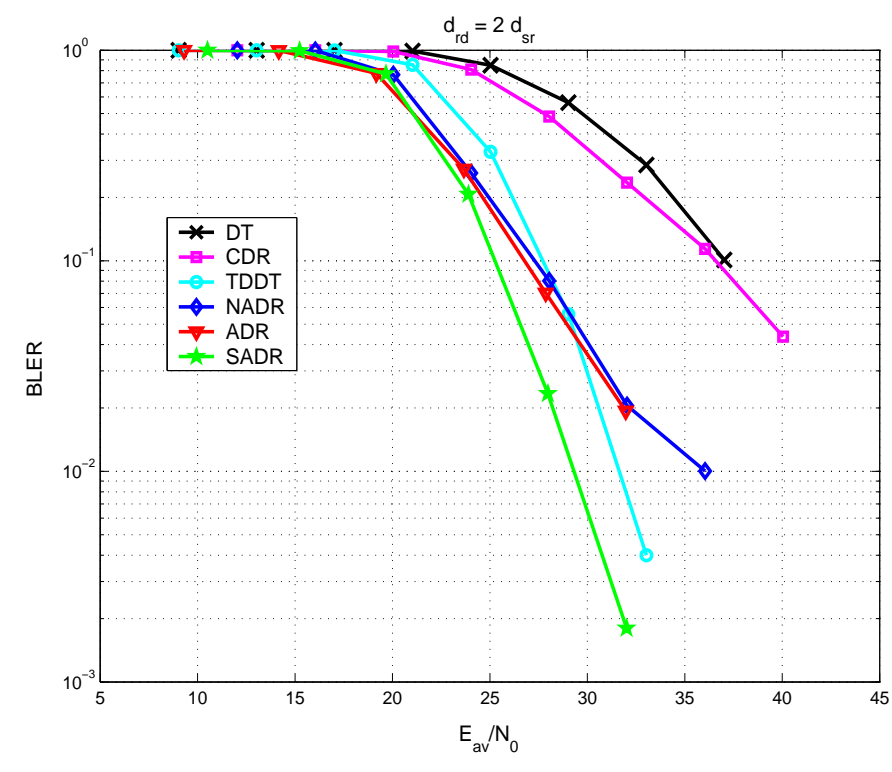

Fig. 4. Block error rates for topology $2, d_{r d}=2 d_{s r}$. Relative channel gains are $g_{s r}=0 d B, g_{r d}=-9.03 d B$ and $g_{s d}=-14.31 d B$

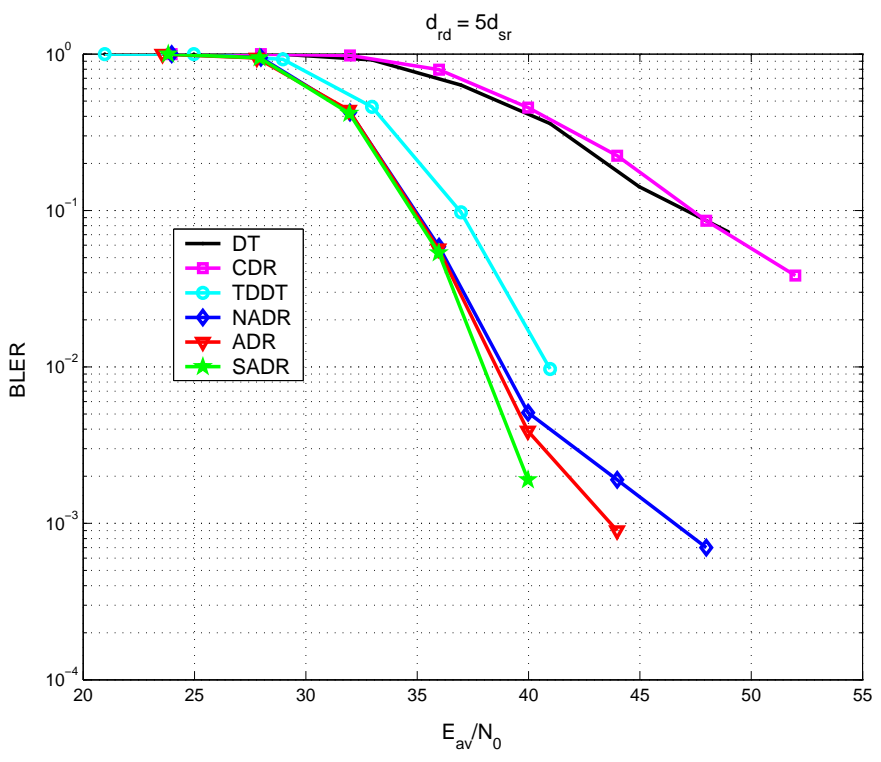

Fig. 5. Block error rates for topology $3, d_{r d}=5 d_{s r}$. Relative channel gains are $g_{s r}=0 d B, g_{r d}=-20.97 d B$ and $g_{s d}=-23.34 d B$

for given source-relay and relay-destination distances. Hence, we expect that the performance gap between the conventional relaying and the diversity relaying schemes to widen for triangular topologies. SADR always outperforms ADR, which proves that the relay can improve the overall block error rate significantly even when it can not detect all of the streams correctly.

We see that the adaptive relaying is more effective on the performance when the average SNR of source-relay channel is close to the relay-destination and source destination channel. As the source-relay channel improves relative to the other channels, all diversity relaying protocols (NADR, ADR and SADR) perform very close.

\section{CONCLUSIONS}

In this paper, we studied digital conventional and digital diversity relaying for spatially multiplexed signals. We proposed two protocols that rely on different levels error detection at the relay. By comparing the performance of all protocols we reached the following conclusions:

- In multi-stream relaying under Rayleigh multipath fading and pathloss, it is worthwhile to use the direct signal even if the source-destination separation is large.

- Adaptive protocols can improve error performance compared to non-adaptive relaying especially when the source-relay channel is not significantly better (in average) than the relay-destination and source-destination channels.

- With linear MMSE combiner as the MIMO receiver, it is useful to allow streamwise decisions at the relay which indicates that two things: First, there are frequent enough occasions where the relay can receive not all but some of the streams correctly. Second, in such occasions, the relay can still help the correct detection at the destination by retransmitting those streams it received correctly.

\section{REFERENCES}

[1] E. Telatar, "Capacity of multi-antenna gaussian channels," European Transactions on Telecommunications, vol. 10, pp. 585-595, Nov. 1999.

[2] G. J. Foschini and M. J. Gans, "On limits of wireless communications in a fading environment when using multiple antennas," Wireless Personal Communications, vol. 6, March 1998.

[3] P. Wolniansky, G. Foschini, G. Golden, and R. Valenzuela, "V-BLAST: an architecture for realizing very high data rates overthe rich-scattering wireless channel," in Proceedings of URSI International Symposium on Signals, Systems, and Electronics, 1998.

[4] V. Tarokh, H. Jafarkhani, and A. Calderbank, "Space-time block coding for wireless communications: performance results," IEEE Journal on Selected Areas in Communications, vol. 17, 1999.

[5] N. Laneman, D. Tse, and G. Wornell, "Cooperative diversity in wireless networks: Efficient protocols and outage behavior," IEEE Transactions on Information Theory, vol. 50, pp. 3062-3080, Dec. 2004.

[6] A. Sendonaris, E. Erkip, and B. Aazhang, "User cooperation diversitypart I: System description," IEEE Transactions on Communications, vol. 51, pp. 1927-1938, Nov. 2003.

[7] J. Boyer, D. D. Falconer, and H. Yanikomeroglu, "Multihop diversity in wireless relaying channels," IEEE Transactions on Communications, vol. 52, pp. 1820-1830, Oct. 2004.

[8] J. Boyer, D. D. Falconer, and H. Yanikomeroglu, "On the maximum diversity order of wireless relaying networks." submitted to IEEE Transactions on Communications, Nov. 2005.

[9] A. Adinoyi and H. Yanikomeroglu, "Multi-antenna aspects of parallel fixed wireless relays." accepted to IEEE WCNC 2006.

[10] Z. Lin, E. Erkip, and A. Stefanov, "Cooperative regions for coded cooperative systems," in Proceedings of GLOBECOM Communication Theory Symposium, Dec. 2004.

[11] H. Bölcskei, R. U. Nabar, O. Oyman, and A. J. Paulraj, "Capacity scaling laws in MIMO relay networks." IEEE Transactions on Wireless Communications, to appear in 2006.

[12] Y. Fan and J. Thompson, "Space-time processing for cooperative relay networks," in Proceedings of IEEE Globecom, Nov. 2005. 